same time the most luminous and the shortest-lived. Consequently, they are ideal for the study of the spiral arm structure of our galaxy; their great luminosity makes them important as distance indicators for other star systems and in studies of interstellar absorption; while their short lifetimes make them sensitive tests of theories of star formation and evolution. In addition, recent work on quasars and blue stellar objects has revealed the depth of our ignorance concerning the distribution of blue stars in the outer parts of our galaxy.

Chapters on spectral classification, photometry, binary stars and spectral analysis are relevant to all types of stars, while that concerning the details of the helium spectrum illustrates the atomic theory of the formation of spectral lines in stellar atmospheres. In the later chapters, several special varieties of early type stars are considered, such as Wolf-Rayet and shell stars, which are as yet little understood theoretically.

With a writer who has herself done so much fundamental research in the field, it is inevitable that personal opinions are sometimes apparent. These, however, only serve to add interest to the book, and to emphasize how much work remains to be done before we have a reasonably complete understanding of the subject. Many of the relevant basic data have been collected and reproduced in tables throughout the book, greatly enhancing its value to the non-expert, and the quality of the diagrams and reproductions of spectra is very high. Only the rather flimsy binding must be criticized, particularly in what should become a standard work. A very thorough list of references completes a book which, in bringing together much diverse information on one topic, should lead to much fruitful research.

R. D. CANNON

\section{OCEAN WAVES AND TURBULENCE}

\section{The Dynamics of the Upper Ocean}

By O. M. Phillips. (Cambridge Monographs on Mechanics and Applied Mathematics.) Pp. vii +261 . (London: Cambridge University Press, 1966.) 60s. net.

THrs elegant book--more elegant than its subject-points to the progress since Ursell's devastating survey appeared in 1960. Many of us will find Phillips's coherent account a very useful guide to some recent work of Longuet-Higgins, Benjamin, Miles and to his own very considerable contributions.

The first three chapters develop the required hydrothermodynamics, allowing for stratification (heat and salt) and rotation. The underlying theory of surface waves includes the simpler non-linear solutions, yielding certain results concerning surface and bottom streaming, the depression of the mean water level over a sloping shelf, and bottom pressure fluctuations in deep water (pertinent to microseisms). The way is now clear for the three main topics: ocean surface waves (eighty-two pages), internal waves (thirty-two pages), and oceanic turbulence (fortyfive pages).

The chapter on ocean surface waves, after some preliminary definitions and discussion of various spectral estimates, gives a systematic and combined treatment of the wave generation theories by Miles and Phillips. The apparatus needed here is substantial, yet I am not convinced that the age-old problem of how the wind generates waves is now essentially solved. In comparison, the subsequent discussion of the saturated frequency spectrum $\left(\sim n^{-5}\right)$, a concept introduced by Phillips, is far simpler and far more rewarding. The role of ripples is emphasized, and the effect of the waves on the wind (profile and drag) receives attention. The chapter closes with a discussion of departure from Gaussian statistics.

The chapter on internal waves introduces into the study of higher modes some of the statistical considerations which have been so rewarding in the study of the lowest (or surface) mode. Approximate solutions are derived for the cases that the wave lengths are long or short as compared with the characteristic thickness of the thermocline. In the short wave limit the presence of a weak shear will twist the wave so as to produce an equilibrium spectrum $\sim n^{-3}$. In the long wave limit the self shear and stratification conspire to produce spectral densities $\sim n^{-1}$ or $n^{-3}$ according to whether the thermocline depth is small or large relative to wave length. The arguments that lead to these relations are fascinating, and the results can be reconciled with observations, but far more definitive observations are required to put these developments on a sure footing. The last section gives a very nice compact survey of the combined effects of stratification and rotation. The co-ordinates in figure 5.10 reveal the author's native hemisphere and discriminate against his northern hemisphere readers.

The final chapter on oceanic turbulence reviews the fundamentals of statistical turbulence theory. It is pleasant that the best confirmation of the Kolmogorov prediction derives from oceanographic measurements (Grant, Stewart, and Moilliet). A principal task here is an assessment of the limitations and modifications imposed by stratification on the isotropic theory. The Monin-Obukhov and Ekman depths define various pertinent layers, and the wave number $\varkappa_{b} \sim N^{3 / 2} \varepsilon_{0}{ }^{-1 / 2}$ separates the inertial from the buoyancy sub-range in the spectrum. Oceanic measurements bearing on these central concepts simply do not exist. In the closing discussion of entrainment and thermocline formation the lack of definitive measurements is even more limiting.

The author's choice of words is delightful: he speaks of "parasitic capillaries", of "foam patches marking the demise of crests that had previously broken", of "the strong, promiscuous interactions" of the turbulent Fourier components, contrasting with "the weak, selective interactions" of the internal gravity waves.

The book emphasizes how much has been accomplished in developing a formalism appropriate to the messy, symbiotic processes in the oceans; yet how little in measuring the pertinent parameters with adequate spacial and temporal resolution. I find the title "The Dynamics of the Upper Ocean" to be at once too specific and too general: too specific because so much of the last two chapters is pertinent to the abyssal oceans; too general because the dynamics of upwelling, of the general circulation, boundary currents, planetary waves is lacking. There is virtually no overlap with Stommel's book on the Gulf Stream, which also deals with ocean dynamics. Phillips's emphasis is on perturbation methods pertinent to ocean processes, and here his original and coherent account is a most welcome contribution.

W. Munk

\section{RADIATION BELTS REVIEWED}

Radiation Trapped in the Earth's Magnetic Field Edited by Billy M. McCormac. (Proceedings of the Advanced Study Institute held at Chr. Michelsen Institute, Bergen, Norway, August 16-September 3, 1965.) Pp. xiv +901. (Dordrecht : D. Reidel Publishing Company; New York : Gordon and Breach, 1966.) \$45.

Thrs Advanced Study Institute was held seven years after Van Allen's discovery of the radiation belts, and the Proceedings disclose and describe the vast amount of information concerning the belts that has been obtained in that time. Particle measurements already represent a substantial survey, covering essentially the whole spatial region of trapping. In energy there is one important gap for protons below $100 \mathrm{KeV}$. It is expected that such protons are the most important source of the "ring current", which is jargon for the current causing the main phase of storms. The important temporal variation is that of the solar cycle and, whereas the original dis- 